Gutiérrez, J. A., \& Concha, J. R. (2014). Modelo gerencial de la empresa exportadora colombiana: análisis de decisiones, estrategia y geoconocimiento. Revista Lebret (6). Bucaramanga, Colombia: Universidad Santo Tomás, pp. 323-347. ISSN 21455996.

\title{
Modelo gerencial de la empresa exportadora colombiana: análisis de decisiones, estrategia y geoconocimiento*
}

\author{
Management model of the Colombian exporting company: \\ decision analysis, strategy and geo-knowledge
}

Jahir A. Gutiérrez O. ${ }^{l}$

José R. Concha V.2

\begin{abstract}
Resumen
El artículo analiza la concepción del modelo gerencial exportador de las empresas colombianas. La incursión empresarial colombiana en diferentes países, en el marco del concepto y escenario de las multilatinas, ha dejado en perspectiva el carácter y la cualificación de la gerencia empresarial internacional del país. Es importante exponer el alcance de la gerencia empresarial internacional global y revisar el carácter propositivo del concepto "multilatino" en el marco de la gerencia para, finalmente, establecer los parámetros del modelo gerencial exportador colombiano. Se destacan la toma de decisiones, el marco estratégico y el amplio geoconocimiento.
\end{abstract}

Palabras clave

Estrategia, empresas multilatinas, geoconocimiento, modelo gerencial exportador.

Códigos de clasificación JEL: M1, M11

\begin{abstract}
The article discusses the concept of management exporting model of Colombian companies. Colombian business break in into different countries, under the concept and scenario of the multi-latins has evidence in perspective the character and qualifications of international business management in the country. It is important to present the scope of the overall international business management and review the proactive nature of the multilatin concept in the framework of management to finally set the parameters of the Colombian exporting management model. Decision making, the strategic framework and broad Geo-knowledge standout.
\end{abstract}

Keywords

Strategy, multilatins companies, geo-knowledge, exporting management model.

Este artículo es producto del proyecto de investigación "Modelo gerencial de la empresa exportadora colombiana. Casos Antioquia, Atlántico, Cundinamarca y Valle; de los grupos de investigación Gestión Empresarial de la Universidad CES de Medellín, Perdurabilidad Empresarial de la Universidad del Rosario, Bogotá y Competitividad y Mercadeo de las Organizaciones de la Universidad ICESI, Cali, Colombia

1 Doctor en Administración Pública. Investigador y Líder del Grupo en Gestión Empresarial de la Universidad CES, Medellín, Colombia. Correo electrónico: algutierrez@ces.edu.co

2 Doctor en Negocios. Jefe del Departamento de Mercadeo y Negocios Internacionales y director del Consultorio de Comercio Exterior (ICECOMEX) de la Universidad ICESI, Cali, Colombia. Correo electrónico: jrconcha@ icesi.edu.co 


\section{Introducción}

La identificación del perfil gerencial del empresario colombiano a escala internacional, es una apuesta que viene articulando el análisis académico, intelectual e investigativo en el presente. El conato de empresas multilatinas ha terminado por exponer una forma de gerencia particular de los empresarios latinoamericanos. De paso, implica reconsiderar los conceptos y planteamientos de gerencia hasta ahora advertidos y, finalmente, extiende la posibilidad para establecer fundamentos propios en la materia. Así, se ha pasado de la revisión externa del concepto a ilustrarse conforme a la región.

La gerencia internacional ha estado caracterizada por los argumentos, criterios y publicaciones de origen anglosajón y europeo, que han servido para el reconocimiento de una identidad general y propia del concepto, de la mano con la administración y la gestión. No obstante, la trayectoria de las empresas ubicadas en dichas áreas geográficas del mundo, habla de un conglomerado de organizaciones que gozan del perfil multinacional (oligopolios) y transnacional (monopolios), estructuras que han terminado por extender el foco de atención hacia la forma de gerencia que desarrollan en estos.

El afianzamiento de la cultura organizacional en las empresas latinoamericanas y el interés por acoger los fundamentos de la gerencia internacional, han terminado por orientar su propio perfil. En consecuencia, la denominación de empresas multilatinas o referencias semejantes hacen alusión al avance en la gerencia de los países de esta región. Este escenario ha impulsado la llegada de las empresas latinoamericanas no solo entre sus pares del continente, sino que han terminado por abrir puertas y afianzarse en el mundo anglosajón y europeo, internalizando los logros adquiridos frente a la gerencia.

El poder determinar los componentes, elementos y fundamentos en que se apoya la gerencia internacional del empresario latinoamericano y por ende colombiano, es una tarea que recoge las inquietudes sobre el arte perdido de la gerencia o su estado del arte, de la teoría de la firma o del empresario innovador o el mismo empresarismo. De ello, se ha superado el grado de dependencia de los centros de conocimiento, gestión e innovación en materia de gerencia, para terminar siendo ubicados como una asignatura de interés profesional en los principales centros académicos y empresariales del mundo.

La estructura sobre la cual se desenvuelve el contexto del artículo responde a los siguientes fundamentos: 1. Análisis sobre la estructura y evolución de la gerencia hasta la vigencia; 2 . La evaluación de los principales métodos y modelos gerenciales contemplados e implementados; 3. La definición del modelo gerencial de la empresa exportadora de las multilatinas; y 4. La concepción de la gerencia exportadora latinoamericana conforme a la definición del modelo establecido. En esencia, la gerencia continúa evolucionando, y con ello, afianzando el carácter de arte que ostenta. 


\section{Organización y evolución de la estructura gerencial en el contexto de las empresas}

La conformación, estructura y evolución del mundo gerencial en las empresas obedecería primigeniamente al periodo entre guerras de los países. No obstante, dicha simbiosis permitiría la consolidación de estructuras empresariales fuertes y de paso, la erudición del concepto de competencia, lo que seguidamente daría lugar a los cimientos básicos de la gerencia empresarial. Esta, básicamente recogería los criterios bélicos en cuanto a estrategia, táctica y toma de decisiones para internalizarlos en sus actividades. La empresa comenzaría el proceso de adaptación incipiente hacia el concepto (figura 1).

Figura 1. Modelo clásico de gerencia

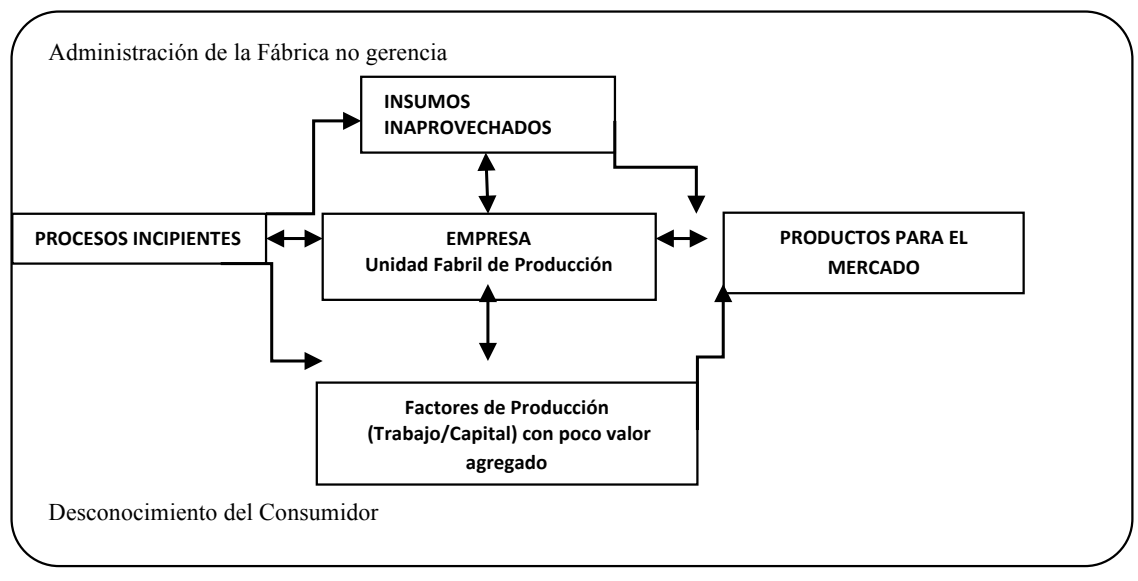

Fuente: autor.

El lugar adquirido por el Estado de bienestar que dejaría atrás al Estado clásico o gendarme, habilitaría a las empresas para ubicar en sus cimientos el análisis científico de la administración y por ende de la gerencia. Así, la máxima de la burocracia, la composición organizacional y la estructura empresarial, serían en adelante los parámetros por considerar para la concepción del concepto gerencial, que para entonces, obedecía más a un orden vertical en el que las jerarquías, mandos y órdenes, caracterizaban el entorno empresarial. Al paso de la institucionalización de dicho Estado se ubicaría la gerencia (figura 2). 
Figura 2. Modelo de gerencia en el bienestar

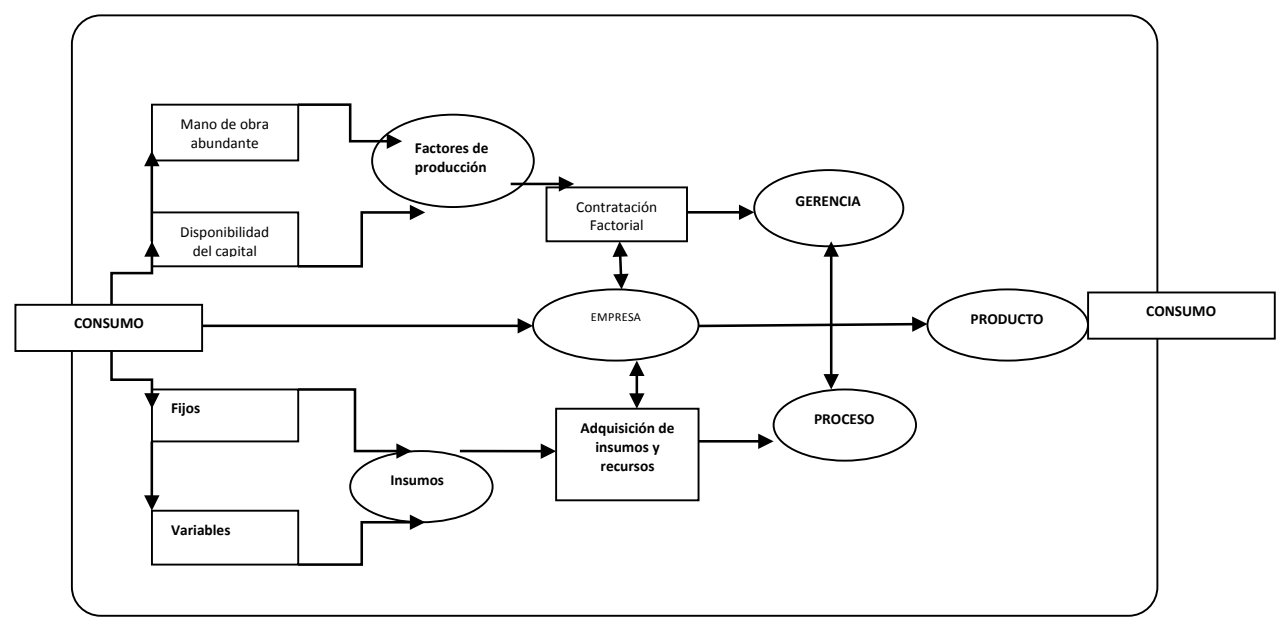

Fuente: autor.

La escasez de recursos condujo para que emergiera con fuerza la apertura comercial y de libre cambio entre los países, epicentro de la posterior internacionalización de las economías. La redimensión de las multinacionales y trasnacionales como fenómeno empresarial plausible, alentaría el interés en las empresas por reconocer en el sector externo una fuente importante de recursos. El mundo empresarial comenzaría a analizar, estudiar y proponer formas de hacer gerencia, a partir del éxito alcanzado por dichas formas empresariales, que posibilitarían en esencia, la aparición del mundo gerencial, argumentan Fernández y Nieto (2002, p. 2):

La internacionalización es la estrategia más compleja que puede abordar cualquier empresa. Pese a tal dificultad, la creciente globalización de los mercados probablemente la haga cada vez más necesaria, incluso para las empresas familiares que habitualmente se han centrado en sus mercados domésticos.

El apoyo económico y financiero por parte de los países hacia sus empresas de origen multinacional y transnacional no se hizo esperar. La inyección de capitales e inversiones provocaría la aparición del mercado financiero internacional y, por ende, el reconocimiento de diferentes fuentes de recursos que sustentarían la apertura comercial inicial y posteriormente económica, tanto de empresas como de países. Así, la libertad económica y empresarial sería el nuevo baluarte de la emergente gerencia internacional en las empresas, ejercicio que terminarían siendo ejemplo para seguir por lo general (figura 3). 
Figura 3. Modelo abierto de gerencia

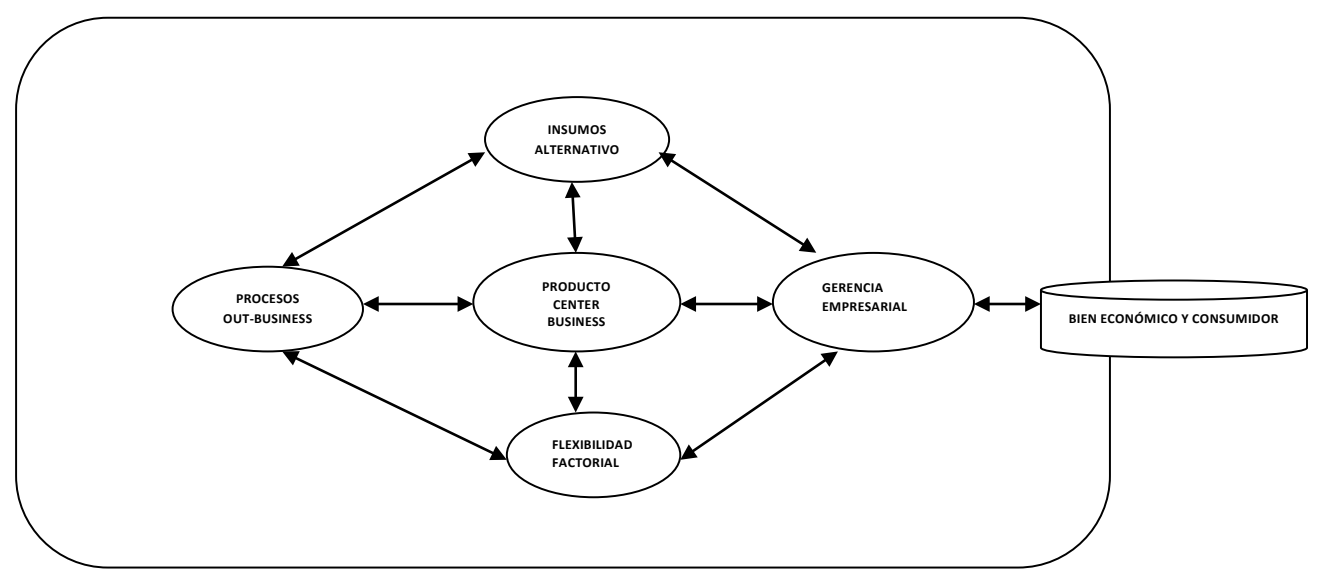

Fuente: autor.

La incertidumbre por la oferta de los recursos y la sostenibilidad del mercado internacional, sumado a los desequilibrios económicos presentados en diversos Estados, terminaría por enfocar la atención hacia la flexibilidad o relajación de las economías, dando paso a la privatización y el desmantelamiento del Estado interventor. Así, lo que hasta hace poco constituyó el mundo empresarial estatal, conocido como sector público, estaría adelante en disposición del contexto privado, prólogo que abriría paso a la gerencia con la toma de decisiones, en la que los empresarios tendrían el carácter de cabeza visible, justifica Nieves (2001, p. 122):

Mientras más inteligente es una empresa y más conocimiento acumula, mayor es la posibilidad de lograr ventaja frente a los competidores del mercado. Las empresas que se aprovechan al máximo sus conocimientos no tienen que repetir tareas, ni perder tiempo en realizarlas, están preparadas para mostrar su rentabilidad, para compartir y no acaparar el conocimiento en la organización. Evolucionan en un espacio propio, tienen la capacidad de conducirse con la efectividad requerida y se desarrolla tanto dentro como fuera de la organización.

El proceso de liberación económica plena, auspiciado por la firma de tratados de libre comercio, incentivaría el interés académico, intelectual y profesional de los gerentes de las empresas ubicadas en países emergentes. El acercamiento hacia dichas fuentes de conocimiento, estudio y propuestas enmarcadas en la gerencia internacional, harían de la modelación gerencial el contexto sobre el que se embarcarían en adelante, las empresas interesadas en tener un espacio en el frente internacional. Periplo que tendría ascendencia en la medida en que las empresas involucradas alcanzasen el nivel de las homólogas internacionales; explican Domingo y otros (1994, p. 34): 
La apertura económica es uno de los elementos que causó desvíos sobre lo programado por las empresas. Estos cambios de entorno siempre crean incertidumbre y tensión en los actores. No obstante, la apertura económica aparece como un proceso de alta credibilidad hacia el futuro, a juzgar por el hecho de que se cita en forma persistente como uno de los tres elementos más importantes por tener en cuenta para fijar la estrategia futura por parte de las empresas.

Es el gerente quien destaca el comportamiento gerencial de las empresas por encima de la formación, preparación o reconocimientos. El propósito de rescatar la capacidad gerencial en distintas latitudes, es lo que ha conllevado precisamente a que se atiendan distintos focos de reconversión relacionados como el referido a la gerencia de las empresas multilatinas o en iguales términos, el relacionado con el mundo empresarial asiático y su lado gerencial. En la actualidad es más complicado cosificar el concepto gerencial a escala de la formación profesional, cuando los empresarios dan cátedra con su gestión (figura 4).

Figura 4. Modelo de gerencia siglo 21

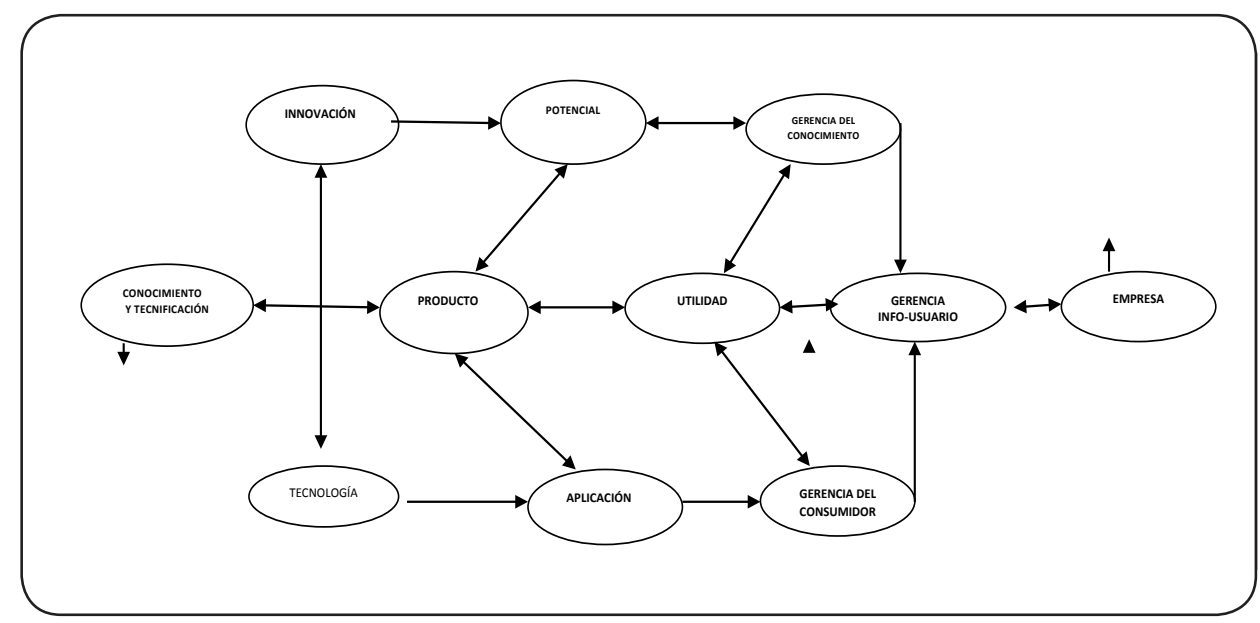

Fuente: autor.

En la actualidad la gerencia depende de la sagacidad del empresario, acompañada de una alta dosis de conocimiento y formación equilibrada con una profunda dimensión en los campos de la innovación y el desarrollo de productos que pasan a ser compulsados por el consumidor y, no una adquisición de este. Para este caso, la gerencia pasa a enfilarse como parte del producto y no como decisor de las acciones de la empresa, para que este sea visible o entregable como hasta hace poco se tenía acostumbrado. La gerencia del siglo 21 está más dependiente del gerente que de su concepto, sintetiza Rodríguez (1995, p. 4): 
A la gerencia interinstitucional que se requiere dentro de cada país, vendría a agregarse una gerencia intrabloques y una gerencia interbloques. En la primera, los "hilos gerenciales" se extienden fundamentalmente hasta los linderos de los países que forman la comunidad económica, mientras que en la segunda, se alargan hasta llegar a los confines del planeta, integrando así todas las asociaciones de países. Por ello se suele hablar del gerente global, que vendría a eslabonarse estrechamente con los gerentes nacionales o regionales y con los gerentes funcionales a nivel mundial, formando así una vasta red de especialistas/generalistas que encararían la administración en todas sus escalas.

Se llegó al punto de buscarle apellido a la gerencia para que de esta pudiera descifrarse la tendencia o el aplomo sobre el que estaban dirigiéndose las organizaciones. De suerte que, la personificación del control, la dirección, la planeación, el justo a tiempo, la estrategia, la reingeniería y la innovación pasan al frente, pero esta vez, trabajando simultáneamente para la empresa. El gerente, desde su comparecencia en el tiempo, ha sido un evaluador de la realidad, la cual le ha servido para compulsar las razones que implican un cambio en el rumbo de las empresas y que claro está aporta cambios relevantes en la concepción de la gerencia, explica Acosta (2004, p. 1898):

Los profundos cambios generados por el proceso de globalización han ejercido fuertes presiones para modificar la forma de dirigir a las empresas $\mathrm{y}$, particularmente, la capacitación y formación de quienes las dirigen, sus gerentes, a los cuales se les exige mayor flexibilidad, rapidez y creatividad en la toma de decisiones.

La preeminencia de empresas multinacionales y transnacionales constituiría el primer encuentro con la administración empresarial internacional (indicios de la gerencia internacional). No obstante, la representatividad del administrador al mando, no ofrecía claros fundamentos para ubicarse en la trama gerencial. En principio, el dueño de la empresa o fundador terminaba representando todo el componente de esta, seguido del sustento sobre el cual fundamentar las explicaciones con respecto al comportamiento de la conducta empresarial.

\section{Análisis y evaluación de los métodos y modelos gerenciales aplicados en las empresas}

Los elementos que han servido para conceptuar acerca de la gerencia han rodeado a esta, hasta el punto de conducirla a una serie de matizaciones que han sido utilizadas para revestir conceptualmente los fundamentos en que se cierne el concepto de gerencia. Unos apuntando a análisis empíricos, categorías, definiciones teóricas o nominaciones que inyectan cualidad o valor pronunciable de lo que significa la gerencia. Cada momento termina por abonar de manera apelativa para sembrar el nacimiento de una nueva definición de gerencia, desvirtuando su esencia corporal en las empresas, reflexiona Ceballos (2004, p. 103): 
El control dentro de una firma es importante ya se esté hablando de una firma doméstica o una firma internacional. Esto porque, como se ha visto, el control dentro de las firmas es una herramienta que permite a la alta dirección alcanzar los objetivos propuestos, evaluar el desempeño de sus funcionarios, medir rentabilidad de la firma, e incentivar a los empleados hacia una meta común. El control cobra mayor importancia cuando es una firma internacional, ya que es de mayor tamaño y mucho más complicada de coordinar.

Lo que se obtiene de la gerencia son resultados en los mercados, los negocios o precios, pero no es claro la utilización de estos para sindicar el estilo de gerencia o connotar que es hacia dichos elementos en los que se puede visualizar la gerencia. Esta es introspectiva, tiene cuerpo, pensamiento y carácter, cuyo vehículo es quien hace las veces de director o gerente, no es una partitura de que al aprehenderse las notas es comprendida la tonada. Prolíficas concepciones acusan lugar de privilegio para significar lo que puede acometerse como eventos gerenciales, expresa Rivera (2009, p. 3):

Vemos con agrado cómo cada día nuestros exportadores son más conscientes de los riesgos de la concentración de destinos, y generan iniciativas que bien vale resaltar para buscar nuevos mercados, claro está, asumiendo la mayor parte del riesgo y buscando mecanismos innovadores para llegar a lugares que hasta hace algún tiempo eran considerados imposibles.

El símbolo del empresario orgánico ha sido desdibujado por la premisa de la formación, por encima de la concepción que sobre las empresas o negocios se alerta que debe tener quien asuma las lides empresariales. La formación es solo un camino de holgura pasiva en donde la empresa solo tiene cabida cuando la formación profesional acaba. Por ello, las empresas son cautas al nombrar líderes recién graduados o sin conciencia visceral sobre lo que implica recibir a cargo una empresa y, más aún, entenderla como un orden biológico con carácter sistémico, expuestas a cualquier asomo de complejidad, contextualizan Hagemeister y Lertxundi (2007, pp. 35-36):

En los negocios internacionales, las empresas deben diseñar su estrategia en entornos muy heterogéneos que las obligan a considerar distintas presiones. Entre ellas, cabe destacar, por un lado, la relativa a la eficiencia global y por otro, la relativa a la sensibilidad local. Cada empresa deberá adoptar la estrategia internacional oportuna (internacional, global, multinacional y transnacional) en función de los parámetros que considere apropiados.

Las empresas requieren que quien asuma su dirección tome la decisión de convertirse en reducto de dicha composición orgánica, y no quien la observa en los elevados pisos de decisión. El gerente debería integrar de nuevo el sentido completo de la empresa en cuanto a la correspondencia con la gerencia. Pasajes de trascendencia privilegiaron la preexistencia de empresarios orgánicos con fundamentación sistémica, que hoy hacen parte de la historia empresarial o de la difuminada clase de dibujar la acción de un empresario representativo con el vacío del ejemplo verbal 
o la pasajera idealización de sus ideas, al respecto señalan Montilla y Melero (2008, p. 14):

El paradigma de la gerencia tradicional comienza a desvanecerse y con ello el sistema de ideas que lo soportan. Hoy la gerencia debe estar enmarcada dentro de las nuevas realidades y tendencias infocomunicacionales. Lo que marcará la diferencia entre un gerente tradicional y el gerente que requiera la sociedad del siglo XXI será indiscutiblemente su forma de aproximarse a la información y el conocimiento: para los primeros seguirán siendo herramientas para producir beneficios económicos, para los segundos algo superior, más asociado a la comunicación y al entendimiento humano y social.

La renombrada preparación a la cual deben trasegar quienes funden sus intereses en la función empresarial, debe reconocer que este solo es un paso posiblemente individual y colectivo, del cual es elocuente el llamado para que el registro de la empresa no sea parte de su vida, sino la vida misma. La aseveración está dirigida a perpetuar el sentido lógico de la vena empresarial, que como casi todas las artes o disciplinas requiere identidad y un alto grado de entrega, pero no de sacrificio, porque se requiere que el líder disfrute de lo que tiene al frente: su empresa, argumenta Pallares (1997, p. 18):

La empresa se concibe bajo este enfoque como un complejo sistema adaptativo, en proceso de aprendizaje permanente, que va desde la adquisición de la información, la utilización de la misma para redefinir sus funciones de manera permanente, modificar sus estrategias y fomentar una mayor fluidez en sus flujos de negocios.

La internacionalización sirve de referencia para que los empresarios reivindiquen la faceta biológica y sistémica de las compañías, representadas con prelación por empresarios que no solo constituían la cabeza visible de las empresas sino que eran la empresa en sí misma, pero igual, cuando se mediaba del centro de poder hacia abajo permeaba en cada espacio de las empresas el adherente papel que cumplía cada directivo o empleado empeñados para que la actividad empresarial tuviese como eco más que el sentido de pertenencia la apropiación directa por todos los que se hacían visibilizar como empleados de acción y no de lema; por lo que justifican Fuentes y Avendaño (1998, p. 42): "A medida que las actividades de comercio exterior se van desarrollando y están creciendo, aumentará también las responsabilidades del gerente de exportación, lo cual implicará un mayor nivel de coordinación interna en la empresa".

La intensidad en los desarrollos que sobre conocimiento e innovación puedan lograr un mayor producto, son las preocupaciones que rondan a la nueva gerencia empresarial, que claro está responde a los mercados, pero no con la consabida predisposición a los precios sino a la información, interés y requerimientos del consumidor en temas puntuales como la especificación del producto o las bases de su diseño para que el consumidor comprenda que compró, pero igualmente evalúe 
si llena sus expectativas, las cuales ya no dependen de la relación de precios sino de la solvencia, versatilidad o impacto que tiene el producto en la exclamación o sensación de quien lo requiere. Ejemplifica Rincón (2004, p. 21): “La globalización debe propiciar el surgimiento o adecuación de empresas capaces de concebir su desarrollo a escala mundial y formular para ello estrategias globales de operación, comercialización y gestión".

El paso que en adelante tomarían las áreas de las ciencias, la innovación, las técnicas y tecnologías cambió la base de pensamiento preocupado por los mercados, a pensar por el futuro de las empresas desde adentro con respecto a lo que por tiempo fue el centro de atención. La sumatoria de todas las injerencias creadas en la gerencia termina absorbida por una ola que no tiene indicios de ser pasajera. La transformación de las empresas independientes a como se llama su origen partirá del pensamiento permanente que enfatiza la capacidad de creación teniendo como norte las áreas mencionadas. Aportan Pico y López (2006, p. 84):

La conformación de las empresas trasnacionales de los países en vías de desarrollo es viable, si se cumple un conjunto de aspectos interrelacionados en las áreas de tecnología, modernización de instalaciones, adiestramiento y desarrollo del recurso humano, organización, estrategia empresarial, reformas institucionales y estructurales, entre otros.

La idea de recomponer en algo el papel de la gerencia y de quien la encarna, recoge gran parte de la preocupación por el estado y la estructura al que ha sido dirigida ella, conforme más a la manera de denominarla bajo una serie de apelativos iterativos, que aunque reflejan un algo de lo que puede estar aconteciendo con el pulso gerencial, provocaron distanciamiento entre la visibilidad gerencial y la capacidad del gerente para efectivamente estar al frente de los resultados obtenidos por la empresa, recuperando el espacio de extensión entre el quehacer de la organización conforme a la referencia que ella tiene acerca del conocimiento y la innovación.

Bien podría decirse que, cada uno de los elementos que ha sido concebido para explicar el desempeño de la gerencia ha tenido como propósito ubicar el campo de acción u objetivo-meta al que han apostado en aras de refrendar su postura. Diversos autores indican que, parte de esta situación ha sido causada por el afianzamiento de la doctrina y la exposición reiterativa del tradicional pensamiento administrativo, poco oportuna, frente a la realidad del quehacer gerencial, que de forma densa, complejiza el marco de referencias para vincular la gerencia a un quehacer específico, derivado en el estereotipo o cosificación de la gerencia, según Mintzberg (2010, p. 107):

Desde luego, pocos gerentes pueden hacerse cargo personalmente de todos los proyectos de sus unidades, incluso de todos los proyectos clave, pero la sugerencia en alguna literatura de que los gerentes no deberían "hacer" nada -ya que se desprecia "el hacer" como una versión de micro administrar-surge de una visión estéril del trabajo: El gerente en un pedestal, fuera de "contacto" literal, limitado a pronunciar estrategias para que el resto de las personas las 
implementen. Quizá lo anterior funcione bien en un mundo simple. El nuestro, infortunadamente (en realidad, por fortuna), es un mundo desordenado. Así que los gerentes tienen que salir y descubrir lo que ocurre y una manera sensata de hacerlo es participar en proyectos concretos.

El gerente formado en el marco de la comentada evolución tiende a encontrarse o involucrarse en diferentes formas empresariales, en las que su conocimiento y experiencia terminan impulsándolas. En la vigencia, a una escala superior de dicho escalafón se encuentran los asesores o comisionados ejecutivos de las organizaciones (CEO), que además de estar en la cumbre de estas, hacen de la gerencia un ejercicio en constante ascenso. La amplia proactividad de la gerencia ha permitido la aparición de dichos CEO, que han terminado por facultar el quehacer en la cumbre de la gerencia.

\section{Concepción gerente - gerencia internacional de las compañías multilatinas}

La concepción del gerente y la gerencia internacional representa una de las áreas de punta que rodea la investigación académica en administración y otras disciplinas. Al respecto, el reconocimiento del aporte dado por las multinacionales foráneas a las empresas emergentes de los países latinos termina siendo un aporte importante. Seguido, de los aspectos y elementos apropiados por los gerentes latinos para afianzarse en la comunidad gerencial internacional, para terminar en últimas, como un modelo gerencial digno de estudio e intereses investigativos enmarcado en el gerente y la gerencia latina. Indica Díaz (2012, pp. 121-122):

El que la internacionalización de las multilatinas se esté consolidando en los últimos años demuestra el profundo efecto de la globalización en los mercados mundiales y la forma en que Latinoamérica se ha incorporado a esta, dejando un tanto obsoletos modelos que muestran la internacionalización como un proceso gradual a realizarse de a poco a lo largo de varios años o incluso décadas. El comportamiento de la IED como manifestación de la superación de los mercados domésticos para las multilatinas, es un símbolo de la globalización de los mercados y de su efecto catalizador de la formación de multinacionales.

El gerente venido de las principales compañías, empresas y firmas del mundo era considerado bastión de los círculos académicos y profesionales en América Latina. Tal es así, que las lecturas, movimientos empresariales y recomendaciones dadas por estas nuevas fuentes de conocimiento pasaron del reconocimiento a la aplicación, intromisión y puesta en práctica de las compañías del área. Sin lugar a dudas, podría decirse que se llegó a acoger de manera personal el nombre o la referencia de un empresario internacional de renombre, para advertir el grado de solvencia de este en las empresas.

Fleury y Leme (2011, p. 109) llaman la atención sobre el que a principios de los noventa, algunas compañías que habían sido un modelo para el desarrollo 
del management o gerencia empiezan a enfrentar una crisis profunda. General Motors, Sears e IBM establecieron nuevos records en materia de pérdidas. Estas empresas enfrentaron graves errores por el rezago de sus decisiones sobre el diseño organizacional y problemas financieros; en suma

Los cambios que venían ocurriendo en el contexto global desafiaron los modelos de gestión e hicieron necesaria su reestructuración. Siguiendo las mismas tendencias, los gobiernos de los países desarrollados tuvieron que reformular sus políticas de desarrollo, prospectando cuáles serían las industrias del futuro para apoyar a las empresas y ayudarlas a construir sus ventajas competitivas Rivera (2014, p. 18).

La adherencia de las doctrinas, premisas y fundamentos generados por las compañías exitosas, condujo para que en las empresas se considerará que la gerencia respondía a hechos comportamentales. Es decir, dependían del gerente al frente de la compañía o de la adaptación de un modelo gerencial que podría ser útil, si era incubado de manera particular. El grado de atención en la materia fue alto, puesto que de ello, se comenzarían a promover diversos cursos y programas académicos para acoplar las nuevas directrices gerenciales, en las que la mitificación hacia el gerente y la gerencia era crucial, analiza Casanova (2011, p. 30):

Los modelos de negocios, de gestión y de liderazgo que enseñamos en las escuelas de negocios se basan primordialmente en las grandes empresas americanas: Dell, Apple, IBM, Google, Citibank, General Electric, Procter \& Gamble. ¿Son estos modelos válidos para las nuevas multinacionales emergentes? ¿Qué tipo de habilidades necesitarán los ejecutivos brasileños y latinoamericanos para llevar a sus empresas al éxito?

El resultado obtenido por cuenta de las empresas globalizadas del interés planteado, para implementar las directrices previstas en los modelos de gerentes y de gerencia, provocarían igualmente que el gerente y la gerencia latinoamericana empezara a plantear sus propias directrices. Por ello, se pasó de la academia, la formación y la ilustración a emprender el proceso de internacionalización, conforme la comprensión y entendimiento generado por los acuerdos, pactos y tratados de libre comercio, que finalmente pondrían a estas, además de prueba, a evaluación frente a la capacidad de respuesta. Expone Cuervo (2010) que aún a pesar de los progresos de los últimos años en el conocimiento de este tipo de empresas, falta investigar a fondo su situación particular.

La entrada de empresas industriales y comerciales del Estado en los diferentes países, a su vez que la conformación por vía de conglomerados de grupos económicos o holdings, sería el paso básico para que comenzará a ventilarse la incursión en otros de las empresas de origen privado y público latinoamericanas. En ambos frentes, el acompañamiento y evaluación por parte de organismos multilaterales como los respectivos Fondos y Banca de Inversión Multilateral, terminarían siendo encargados de escenificar este tema para que en esencia se tomara dicho asunto 
como un campo alcanzable en sí. Tal como plantea Yákovlev (2013) el fenómeno de la transnacionalización es progresivo y será fundamental para determinar la orientación de los mercados regionales y globales.

El ajuste jurídico tendría su espacio para que las empresas con perfil internacional tuviesen tiempo de acondicionarlas a la medida de las exigencias. Por ello, no es desconocido que el origen de las multilatinas tendría como base la capacidad y condiciones jurídicas para aglutinarse, conformarse y estructurarse en diferentes formas empresariales. Básicamente, la adquisición, compra y enajenación de empresas se convertiría en el punto de quiebre para que aparecieran los recursos con que solventar el apalancamiento, necesarios para auspiciar la incursión internacional de estas.

Tabla 1. Perfil de internacionalización de la empresa

\begin{tabular}{|c|c|c|}
\hline DIMENSIÓN & EXPLICACIÓN & POSIBLES MEDIDAS \\
\hline Ingresos & Internacionalización desde la demanda & Ventas externas totales \\
\hline \multirow{3}{*}{ Recursos } & \multirow{3}{*}{ Recursos ubicados en el exterior } & Activos externos totales \\
\hline & & Subsidiarias externas totales \\
\hline & & Porcentaje de empleados extranjeros \\
\hline \multirow{2}{*}{ Geografía } & \multirow{2}{*}{ Dispersión Geográfica } & Número de regiones o países de operación \\
\hline & & Diversidad en los ambientes externos \\
\hline \multirow[b]{2}{*}{ Actitudes } & \multirow[b]{2}{*}{ Orientación internacional del personal } & Número de gerentes con experiencia internacional \\
\hline & & $\begin{array}{l}\text { Años de experiencia internacional de los gerentes/ } \\
\text { años trabajados }\end{array}$ \\
\hline \multirow[b]{2}{*}{ Capital } & \multirow[b]{2}{*}{ Internacionalización Financiera } & Porcentaje de préstamos externos \\
\hline & & Deudas externas / Total \\
\hline Relaciones & Internacionalización de la Red Empresarial & Número de alianzas y socios internacionales \\
\hline
\end{tabular}

Fuente: Cerrato \& Depperu, 2011. En Díaz, 2012, p. 119.

La empresa multilatina proviene de la flexibilidad que en materia de derecho empresarial, de firmas y de sociedades fue conducida por los diferentes países latinoamericanos, empresarios y organismos de carácter internacional, para que efectivamente se dispusieran los recursos económicos y financieros requeridos para dicho frente de trabajo. A la luz de esta fuente de recursos, el análisis relacionado en cuanto a la manera de acceder, incursionar o exponerse en el frente internacional 
correría por cuenta de los organismos mencionados, los cuales al evaluarlas darían el dictamen final del proceso.

Tabla 2. La más grandes multilatinas por montos de ventas (2011, US\$ millones)

\begin{tabular}{|c|c|c|c|c|}
\hline & Empresa & País & Sector & Ventas \\
\hline 1 & Petrobras & Brasil & Petróleo y gas & 130171 \\
\hline 2 & PDVSA & Venezuela & Petróleo y gas & 102500 \\
\hline 3 & Vale & Brasil & Minera & 55014 \\
\hline 4 & América Movil & Mexico & Telecomunicaciones & 47900 \\
\hline 5 & Grupo JBS Frioi & Brasil & Industria Alimenticia & 32944 \\
\hline 6 & Odelbrecht & Brasil & Constructora & 32325 \\
\hline 7 & Gerdau & Brasil & Metalurgia & 18876 \\
\hline 8 & FEMSA & México & Bebidas & 14502 \\
\hline 9 & Cencosud & Chile & Comercio & 13971 \\
\hline 10 & Cemex & México & Cemento & 13546 \\
\hline 11 & Brasil Foods & Brasil & Industria Alimenticia & 13486 \\
\hline 12 & Grupo Alfa & México & Conglomerado & 13053 \\
\hline 13 & MARFRIG & Brasil & Industria Alimenticia & 11549 \\
\hline 14 & Grupo Bimbo & México & Industria Alimenticia & 10463 \\
\hline 15 & Tenaris & Argentina & Metalurgia & 9973 \\
\hline 16 & Camargo Correa & Brasil & Constructora & 9610 \\
\hline 17 & Falabella & Chile & Comercio & 9044 \\
\hline 18 & Andrade Gutierrez & Brasil & Constructora & 8400 \\
\hline 19 & Telmex & México & Telecomunicaciones & 7057 \\
\hline 20 & TAM & Brasil & Transporte aéreo & 6927 \\
\hline 21 & Grupo Modelo & México & Bebidas & 6539 \\
\hline 22 & LAN & Chile & Transporte aérero & 5718 \\
\hline 23 & Votorantim Cimentos & Brasil & Cemento & 5680 \\
\hline 24 & Sudamericana de Vapores & Chile & Transporte marino & 5152 \\
\hline 25 & Embraer & Brasil & Aviación espacial & 5141 \\
\hline 26 & Brighstar & Bolivia & Telecomunicaciones & 4700 \\
\hline 27 & Grupo CASA SABA & México & Comercio & 4670 \\
\hline 28 & CMPC & Chile & Maderera & 4613 \\
\hline 29 & Grupo Televisa & México & $\begin{array}{l}\text { Corporación de comunicación } \\
\text { masiva }\end{array}$ & 4487 \\
\hline 30 & Arauco & Chile & Maderera & 4451 \\
\hline
\end{tabular}

Fuente: América Economía, 2012. 
El origen de las multilatinas contempló un alto contenido técnico a la hora de llevar el proceso. No solo fueron considerados los fundamentales macroeconómicos o de política económica internacional, la revisión en detalle de la capacidad y naturaleza de las empresas latinas para percibir el impacto del sector externo fue apreciado a cabalidad. Por ello, puede señalarse sin lugar a dudas, que la aparición de estas estructuras fue atendida bajo la mayor rigurosidad, y que por ende, la extrapolación de las fronteras terminaría siendo un aliciente más que un resultado, a la justa medida de la evaluación hecha. Señalan Revuelta y Alonso (2013) que el fenómeno adquiere un carácter significativo, a pesar de su reciente iniciación.

Es importante establecer el impacto generado por las multinacionales y transnacionales foráneas en el activismo multinacional desarrollado por las compañías multilatinas. Se ha pasado de la revisión de las experiencias, modelos y resultados de las empresas foráneas, a ser revisados los casos latinoamericanos. Así, las empresas y gerentes de compañías multilatinas se establecen como modelos gerenciales de paso, la ilustración de ambos se hace necesaria para identificar el proceso, para finalmente, reconocer el alcance que puede tener la concepción gerencial en la región. Cuervo (2007) hace notar que solo hasta los años noventa del siglo pasado las multilatinas empezaron a posicionarse en los mercados internacionales en contraste con las de los países asiáticos, como Corea del Sur o Taiwán en donde el fenómeno se inició veinte años atrás.

Ha sido relevante el alcance obtenido por las firmas de distintos sectores provenientes de Latinoamérica, que impulsadas por diversos intereses han terminado por ser ubicadas en el rango de empresas para seguir. La distinción no solo ha permitido replantear las aseveraciones vinculadas al bajo valor agregado generado en ellas, sino que además, han terminado por transferir parte de su capacidad instalada y equipamiento a los países de los cuales hasta hace poco, la acción gerencial giraba parcial o totalmente entorno a ellos con respecto a la adaptación de modelos, formación profesional y resultados. Revuelta y Alonso (2103) resaltan la dificultad de establecer con exactitud las características de las multilatinas cuando se trata de análisis acotado como el que intentamos en este artículo.

La gerencia empresarial internacional destaca el papel que cumple quien al frente de las compañías considera a la economía y al mercado fuera de las fronteras tradicionales, como una oportunidad para fortalecer las perspectivas de la organización. Temática que ubica a la gerencia en el relevante rango de jugador multinacional, capaz de fundar o promover lazos de expansión de la empresa en diferentes frentes relacionados con las opciones productivas, o incluso, refundar la organización conforme a criterios basados en expansión, fortalecimiento o consolidación fruto de adquisiciones o fusiones. Expone Botello (2014) que el actual es un momento apropiado para incentivar en este tipo de empresas transformaciones en términos de infraestructura, estrategias empresariales y políticas de internacionalización que defina una verdadera reconversión productiva de América Latina. 
La evolución gerencial ha permitido contemplar diferentes espacios de actuación para esta, gracias a la conducta empresarial, al pasar de cerradas a competidoras o completamente abiertas a la actual segmentación. La constante derivación de marcos gerenciales reivindica su evolución, a la par, con la apertura para que aparezcan alternativas como las denominadas multilatinas, para finalmente, plantear para el caso colombiano, la concepción del modelo gerencial empresarial exportador desde dichas bases. En esencia, el arte de la gerencia aún tiene espacio para otros frentes de análisis. Establecen Castro y Jaller (2012, p. 49) que:

From the top 30 Multilatinas according to the Journal Américaeconomíajournal, the most important Multilatinas are from Brazil (10), Mexico (8), and Chile (7), followed by Argentina (3), Bolivia (1), and Peru (1). In fact, we realized that Brazil, Mexico, and Chile are countries that have led the process of foreign direct investment of Latin America. Due to the particular conditions in which Multilatinas have emerged, we found that these companies are more flexible and adaptable than traditional MNEs. For this reason, Multilatinas have been dubbed "unconventional multinationals" owing to the fact that they are more open, creative, and innovative. Nevertheless, we suggest that if Multilatinas want to stay competitive in the national and the international arena, they will have to invest in R\&D and technology in order to add value to their products.

El fenómeno empresarial latinoamericano tendría finalmente su aparición a comienzos del siglo 21, en el que la gestión y gerencia abrirían espacio en empresas emblemáticas de orden nacional, que terminarían saltando al alcance de las economías y los mercados internacionales. En la actualidad, la tendencia de las empresas y los gerentes de esta área del continente americano está dirigida a la consolidación de una serie de proyectos de adquisición, inversión y proyección de nuevas empresas en diferentes países del continente, que han terminado por destacar la avanzada gerencial latinoamericana.

\section{Definición e ilustración del modelo gerencial de la empresa exportadora en Colombia}

La gerencia empresarial se encuentra orientada por las disposiciones surtidas por la composición de las organizaciones y la manera cómo estas han percibido y puesto en marcha las condiciones del mercado y de las economías a favor de las empresas. El rótulo de empresas exitosas descansa, precisamente, sobre el rango en el que ha terminado consolidada por cuenta de quien está al frente de la empresa, revestido con diferentes designios que en suma no logran contextualizar concretamente lo que significa la gerencia o si esta es un paso por cuenta de etapas que de cierta manera deben recorrerse. Expresan Devlin y Moguillansky (2009) que la incapacidad de algunas empresas para evaluar adecuadamente el entorno en busca de oportunidades y limitaciones se explica por una combinación de miopía empresarial y externalidades o fallas de los mercados. 
El acompañamiento que sobre la gerencia empresarial ha tenido la movilidad de los Estados nacionales ha sido relevante. Cada paso dado por las instituciones económicas y políticas de los países han tenido efecto directo en el quehacer empresarial. De allí que, las empresas hayan tomado para sí las disposiciones hechas en las líneas estatales para incorporarlas a su propio ejercicio. De manera simultánea, cada cometida elaborada en la estructura estatal tendría como recibo a la empresa para acomodarla en su composición organizacional. De ello, claro está, la gerencia sería un lineamiento crucial. Tal como plantean Ferrer y Clemenza (2006) el posicionamiento competitivo de la empresa depende de su actitud creativa ante la incertidumbre que marca los entornos actuales y de su capacidad de desarrollar competencias distintivas que le permitan enfrentar la compleja competencia del mundo global.

Las diferentes derivaciones a las que ha sido llevada la gerencia son prueba fidedigna de los elementos con los cuales se ha querido explicar las amplias y variadas ramificaciones a las que está sujeta la gerencia, siendo la liana internacional lineamiento de los filamentos que debe ir acogiendo, adaptando y poniendo en marchas las empresas. En particular, de cierta manera no es fácil descifrar dicha composición por cuanto existen otros elementos, además de los hechos mediáticos, que recrean las bases para sustentar los criterios que explican el comportamiento gerencial internacional. En la perspectiva de Hamel y Välikangas (2003) la competencia incentiva el desarrollo de las potencialidades de la empresa y su capacidad para adaptarse a las nuevas condiciones.

La ruta de modelos adquirida con el paso de la ubicación de más derivaciones contenidas en el sustrato de elementos que acompañan a la gerencia, de cierta manera, han terminado por fraguar elementos que pueden desviar la atención sobre el ejercicio gerencial. Son notables los procesos de dilación que pueden recibir este y otros temas, cuando a la parte de las consecuencias se pretende agregar más acervo a su concepción, cuando en realidad continúa siendo una, la gerencia, observada en diferentes momentos. Es la gerencia internacional empresarial un momento que la explica en la actualidad.

Es la oportunidad de extender la posibilidad de las ramificaciones, el aprendizaje y conocimiento que con respecto a la gerencia convalida los criterios aislados y separados con los cuales se pretende componer la idea de que la gerencia es compleja, pero con simultaneidad de tareas que no necesariamente son conexas. La realidad es que la gerencia está supeditada a responder a diferentes aspectos que aún no colman la composición de los manuales o estudios vinculados a la gerencia, que es propia de quien está al frente de los designios de la empresa, y que no se alcanzan a estimar totalmente; a propósito plantea Villareal (2005 p. 69):

En el complejo entorno global en el que actualmente se desenvuelven las empresas, la gestión empresarial internacional posibilita comportamientos y actuaciones que, prácticamente, especifican a cada organización, haciendo más 
compleja su caracterización y generando un altísimo grado de heterogeneidad entre las empresas internacionales. Desde esta perspectiva, hemos tratado de resolver ciertos problemas de ambigüedad que se generan en la delimitación conceptual de la internacionalización de la empresa como estrategia y de la empresa multinacional como entidad organizativa. Este problema de indefinición se explica, fundamentalmente, por el alto grado de heterogeneidad intrínseco a dichos conceptos.

La administración, por su parte, ha servido para que la gerencia sea conjeturada en momentos precisos para la empresa como la toma de decisiones estratégicas, la definición de políticas corporativas o tareas por seguir. En este tramo de las explicaciones ha prevalecido cierto desgaste a la hora de interpretar concretamente las bases en que se apoya la gerencia. Ha sido el interés por confinar la gerencia a tareas específicas y rutinarias, que en muchos casos terminan por extraviar el propósito de elevar la gerencia a los niveles deseados para la trayectoria de la empresa y del gerente al frente de ella. Para Puerto (2010) las situaciones de crisis intensifican la búsqueda de estrategias alternativas para permanecer en el mercado y diferenciarse de la competencia.

El sometimiento a funciones operativas, que podrían caer en otros empleados o funcionarios, dilata la posibilidad de profundizar el discurso y formato de explicación que rodea a la gerencia, para lo cual los cánones o estereotipos a los que termina siendo sometida, no pueden dilapidar la trayectoria compleja y sistémica que rodea a la gerencia y que con cargo a ella se trata de explicar y hacerse aplicar para quienes atienden los criterios gerenciales como elementos a fondo que deben seguirse. Si bien es necesario establecer las tareas a las que se debe el gerente, la simplificación que estas puedan traer rompe con el hilo explicativo en el que se trata de ubicar e impulsar al gerente. Considera Pérez (2004) que aquellas situaciones en las que la empresa debe generar soluciones a problemáticas nuevas son las más enriquecedoras para el aprendizaje institucional.

El gerente busca además de factores productivos, nuevos mercados y oportunidades de negocios, que se trasladan a la senda de análisis y perspectivas tejidas en los intereses de la empresa. Definir el gerente internacional, parte de la concepción de las actividades a las que debe responder este ante la junta directiva y los accionistas, más que a un acartonado proceso de forma mediática. El margen internacional puede lograrse por efecto económico, jurídico o vía tratados internacionales, tras los que las empresas emblemáticas son las primeras en ser mostradas a la par con el gerente hacedor de ellas.

El valor consolidado en las empresas permite orientar el interés hacia ella, escenario que muestra la realidad a la cual está abocada la gerencia de la empresa. En un momento en el que, las evaluaciones y valoraciones económicas se convierten en un mecanismo fructífero para estandarizar en una línea de trabajo la tendencia de favorecer los factores y variables circunstanciales con la realidad de la empresa de 
cara al proceso de internacionalización que involucra a toda la estructura y no solo al gerente internacional. Amplían Bustamante y Opazo (2004, p. 16):

En los procesos gerenciales se asume que es imposible controlar el ambiente, y ni siquiera tomarlo como poco cambiante es una buena idea, ya que se está frente a la variable generadora de incertidumbre que provocará la mayor cantidad de estados posibles en la estructura del sistema a fin de sostenerla en equilibrio con su entorno.

La formación de quien lidera la organización es necesaria más no suficiente. En cabeza de la gerencia, debe tenerse claro el mapa de alcance, desarrollo y fortalecimiento de la empresa, mas esto requiere de una revisión profunda sobre qué áreas de dicho mapa han de ser localizadas. La posición de compañía relevante transversal, multiproducto o multipaís, hace parte de los elementos para evidenciar los fundamentos locales como internacionales, que en términos generales no son tan erráticos o equívocos entre sí, por cuanto, las finalidades, propósitos y metas se conocen plenamente, pero no su gerencia.

La internacionalización de las empresas no es solo reducto del paso de la frontera local hacia la foránea. Es claro que la vigencia de una empresa extranjera debe ser tal, para que eventualmente puedan establecerse elementos de arraigo en el país de llegada para comenzar a extender la idea de la gerencia internacional. No es donde están los negocios sino lo que representa cada uno en los países en donde se encuentran frente al respaldo que estos generan en términos del fortalecimiento de la gerencia multidestino, con respecto a lo que representa en cada país de manera convergente y diferencial. Para decirlo en palabras de Jiménez (2007, p. 124) "las empresas son el reflejo de sus gerentes", para hacer notar que las decisiones gerenciales inciden directamente en el posicionamiento de las organizaciones.

La talla de la gerencia internacional se logra en la medida que se supera la agrupación o multiplicación de marcas, cuadros organizacionales o estructuras corporativas o la redefinición de conglomerados o grupos empresariales, por unos estándares que permiten plantear cada rubro bajo el carácter de la gerencia internacional. En un proceso de ensamblaje que requiere de lineamientos que revisten el contexto, sobre el cual, la adquisición o compra de empresas es solo un baluarte a la hora de refrendar el proceso. Para Bonomie y Añez (2009) son imperativas, en las condiciones actuales, las relaciones interempresariales para enfrentar los cambios y retos del entorno.

La entrada de empresas a grupos económicos u organizaciones empresariales no avanza más allá de mostrar nuevos negocios o la traslación de capital hacia otras fuentes de recursos. El tono de la estrategia o del fundamento por el cual se toman decisiones similares, hacen parte de la reserva poco visible que refrenda las acciones ocultas de la gerencia. El escenario de probabilidades de reconocer las bases en que se recrea la estrategia, es solo un asunto de información que la organización expresa 
para advertir a los medios de su interés, pero en ningún caso, reiteran la inquietud gerencial. Complementa Quero (2008, p. 47) que:

El papel del gerente debería contemplar en primer lugar, la capacidad de búsqueda del equilibrio indispensable entre la organización y su entorno, lo cual viene a ser una de las responsabilidades fundamentales. En segundo lugar, el gerente debe conducir su gestión hacia la optimización de los recursos que se colocan bajo su responsabilidad tanto en el ámbito tecnológico y de producción, como en el administrativo y humano-social. En tercer lugar, el gerente debería encauzar en la misma dirección, todos los esfuerzos e iniciativas orientados al desarrollo integral de la organización.

El asunto es saber cómo ilustrar un campo que obedece más a la discrecionalidad de quien lidera con la venia de la junta directa y de los accionistas, y de estas últimas, sobre la manera como tratan de evidenciar en un contexto amable o adverso la posición que asume la empresa a la hora de orientar la gerencia internacional. Los contados episodios bajo las movidas empresariales muestran el andamiaje macro cefálico sobre el que está pensada la organización, pero si se pudiera discernir a partir de la estructura el componente gerencial este sería además de un gran acierto, un éxito argumentativo. Según Aubert (2009) las empresas multinacionales han generado procesos internos de continuo aprendizaje y consolidan, así una perspectiva de largo aliento, en tanto en las familiares es difícil encontrar decisiones que superen el análisis coyuntural.

La perspectiva que trae el identificar la gerencia con los pasos que da la organización, muestra la naturaleza lógica de que debe ser este quien reúna todos los argumentos para no solo estar al frente de la compañía, sino para entender de manera profunda los productos a los que la empresa ha dedicado conocimiento e inversión. Aún, no hemos hecho amplia traza de lo que significa ver a un Buffet, Gates, y Jobs cuando asoman en los escenarios para hablar de las nuevas apuestas que sobre innovación están generando sus compañías, y que de ello, cuan relevante es lo creado para mejorar las condiciones de la sociedad o de quien adquiere algún producto de su organización. Argumentan Lanzas et., al (2008) que el conocimiento del estilo gerencial del equipo directivo se constituye, en sí mismo, en una ventaja competitiva de la organización y, adicionalmente, cuando ese estilo se define a partir de un incremento constante de la voluntad y la disponibilidad para atender la misión mediante la disposición de recursos de todo tipo.

Asimismo, una nueva dimensión que enaltece el orden y la comprensión de la gerencia, que la pasa del escenario de los resultados, al epicentro del conocimiento y la innovación, en donde el gerente ya no se reserva el conocimiento que tiene de la empresa, por el contrario, lo recrea y lo estipula como directriz sobre la cual debe llevarse el sentido empresarial, escenario sobre el que la innovación deja el lugar de asiento y sustrato, al tomar el primer rubro de balance para definir la proyección empresarial. Indica Labarca (2008, p. 64) que "El sistema de valores está constituido 
por la formulación de la misión, la visión y la filosofía de la organización, y es acordado por la alta gerencia".

La estructura empresarial ha compulsado diversos manifiestos para establecer el quehacer de la gerencia a escala organizacional. Para saber al respecto, es necesario ubicar las condiciones en que se desenvuelve la gerencia en general. Luego, considerar las bases en que se indica que las empresas revisten el axioma del empresario, y el gerente el de la empresa, del cual debe no solamente caracterizarse sino ilustrarse el sendero en los procesos de internacionalización que podría cubrir la empresa y el gerente de cara precisamente a dicha exploración fuera de las líneas habituales de trabajo.

\section{Conclusiones}

La gerencia ilustrada como el espacio en el que se generan las ideas y la dirección que toman las empresas está en la mira de quienes no la conciben un marco estatutario, sino escenario de trabajo en el que se "recrean" diversidad de posibilidades para el bien de las empresas, entre las que cabe la innovación de manera central. El gerente supeditado a la evaluación permanente de su gestión por cuenta del comportamiento del mercado y la producción, ha tomado las riendas de la organización al trasladar la innovación como modo de producción empresarial, y claro está, fuente de la autoevaluación gerencial.

El pasó de diferentes concepciones que han acompañado el ascenso gerencial ha servido para revestir el carácter del gerente. Su diseminación impedía conocer el estado de la cuestión sobre el éxito de la empresa y la responsabilidad que con cargo a ella tuviera la acción y las decisiones tomadas en la gerencia. Tal es así, que llegó a ser convertido en un banco de ejemplos y recetas, condenado a la cabeza de la organización a transformarse en un seguidor del mercado, dejando su carácter de líder y la evaluación, a expensas de la perspectiva competitiva empresarial.

Los temas sobre conocimiento, desarrollo e innovación han valido para cubrir y fundamentar el sentido de la gerencia, en un orden sobre el cual el gerente dentro de la empresa pone a prueba sus ideas, por fuera del marco de juego de roles en las que desde el organigrama se asienta en la empresa. Los preceptos que corroboran esta apreciación son de carácter axiológico e intelectual y acompañan la personalidad del gerente, quien, a su vez, aprovecha sus virtudes, para afianzar el bagaje y conocimiento que ha cumplido en ellas, y que le ha valido alcanzar la connotación gerencial.

Es demasiado relevante para el mercado, que sea el gerente quien ilustre bajo su conocimiento el sentido que entiende por innovación en la empresa y cómo esta hace parte de los desarrollos que se logran para cumplir las metas. El gerente como ordenador de los abordajes de la empresa en materia de innovación, permite 
congraciar la idea acerca de quién en adelante debe resaltar los resultados de la empresa, bajo los preceptos en que sean instituidas las ideas. Si bien, se reitera la contundencia de los datos financieros, estos pasan a depender de los aciertos en el mercado, del quehacer del gerente y las brechas de la innovación empresarial.

\section{Referencias}

Acosta, A., Villegas E., Mavarez, E. (septiembre-diciembre, 2004). La capacitación gerencial en pequeñas y medianas industrias (PYMIs) zulianas. Revista de Ciencias Sociales, X(3), 441-448. Maracaibo.

Aubert, G. (2009). Diferencias en el estilo de liderazgo de los ejecutivos, entre empresas multinacionales que operan en Costa Rica y empresas locales. Gaudeamus, 1(1), 41-63.

Bonomie, M. E., y Añez, H. C. (2009). Reflexión teórica de las estrategias flexibilizadoras en el marco de la globalización. Revista Venezolana de Gerencia (RVG), 14(48), 592-605. Universidad del Zulia (LUZ).

Botello, H. A. (2014). Condiciones y determinantes de la internacionalización de las empresas latinoamericanas. Apuntes, 41(75), 47-78. Universidad Industrial de Santander.

Bustamante, U. M. A., \&. Opazo, B. P. A. (2004). Hacia un concepto de complejidad: sistema, organización y empresa. Serie Documentos FACE SDD, 3(2), 1-21. Universidad de Talca.

Cardona, D. (2010). Industria. Los gerentes y sus habilidades en la empresa. La República. S.v. Alta gerencia, pp. 26. www.larepublica.com.co

Casanova, S. L. (2011). El ascenso de las multilatinas en la economía mundial. La nueva geografía de la internacionalización. ICE, (859), 21-31.

Castro, O. J., \& Jaller, C. I. (2012). Internationalization patterns of multilatinas. Revista Ad-minister, (21), 33-54.

Ceballos, F. N. (2004). Sistemas de control gerencial en los negocios internacionales: enfoques y antecedentes empíricos. Seminario para optar al título de ingeniero comercial mención administración.

Cerrato, D., \& Depperu, D. (2011). Unbundling the construct of firm-level international competitiveness. The Multinational Business Review, 19(4), 311-331.

Cuervo, C. Á. (2007). Liberalización económica y multilatinas. Georgetown University. Journal Globalización, Competitividad y Gobernabilidad, 1(1), 66-86.

Cuervo, C. Á. (2010). Multilatinas. Universia Business Review, 1-33. 
Devlin, R., y Moguillansky, G. (2009). Alianzas público-privadas como estrategias nacionales de desarrollo a largo plazo. Revista CEPAL, (97), 97-116.

Díaz, S. H. E. (2012). Aportes a la evaluación de la competitividad internacional de las multinacionales. Suma de negocios, 3(2), 115-122.

Domingo, R., Pastori, H., y Veraé, T. (1994). Comportamiento estratégico de las empresas industriales frente a la apertura. Documento de trabajo.

Fernández, Z., y Nieto, M. J. (2002). La estrategia de internacionalización de la pequeña empresa familiar. (Documento de Trabajo). Series de Economía de la Empresa, 2(11), 1-27.

Ferrer, J., y Clemenza, C. (2006). Habilidades gerenciales como fundamento de la estrategia competitiva en los sectores de actividad metalmecánica venezolana. Tendencias, 7(1), 81-100. Revista de la Facultad de Ciencias Económicas y Administrativas. Universidad de Nariño.

Fleury, A., \& Leme F., M. T. (2011). Brazilian Multinationals. Competences for internationalization. Cambridge University Press.

Fuentes, C. E. L., \& Avendaño, N. R. (1998). Gerencia de Exportación. Banco Nacional de Comercio Exterior. S.N.C. (1 ${ }^{\mathrm{a}}$ ed.). México: Bancomext.

Hagemeister, M., \& Lertxundi, L. A. (2007). Las estrategias de la gestión internacional: el conocimiento como componente clave para su clasificación. Cuadernos de Gestión, 7(2), 29-37. Universidad del País Vasco.

Hamel, G., \& Breen, B. (2009). El futuro de la administración. (2a. ed.). Harvard Business School Press. Barcelona: Grupo Editorial Norma.

Hamel, G., \& Välikangas, L. (2003). En busca de la resilencia. Harvard Business School Publishing Corporation.

Jiménez, M. H. (2007). Modelo de competitividad empresarial. Umbral Científico, (09), 115-125. Fundación Universitaria Manuela Beltrán. Bogotá.

Labarca, N. (2008). Evolución del pensamiento estratégico en la formación de la estrategia empresarial. Opinión, 24(55), 47-68. Universidad de Zulia. Maracaibo.

Lanzas,Á. M., Arias,O. J., y Castañeda, M. C. (2008). Estrategias gerenciales administrativas para las empresas comercializadoras de productos alimenticios procesados al por mayor. Scientia et Technica, 15(40), 121-130. Universidad Tecnológica de Pereira.

Mendoza, J. M. (2006). Innovación por lo alto. Imaginación y acción en la empresa. ( $1^{\mathrm{a}}$ ed.). Bogotá: Ediciones Asesores del 2000.

Mintzberg, H. (2010). Managing. (2a. ed.). Bogotá: Grupo Editorial Norma. 
Montilla, M., y Melero, R. (2008). Competencias clave del gerente en el contexto de la sociedad de la información. Revista Electrónica de Estudios Telemáticos, 7(1), $1-14$.

Nava, Y. (2008). IV Congreso Internacional de Gerencia en América Latina. Perspectivas Gerenciales en el Siglo XXI. Modelos, Estrategias, Alcances y Retos. Revista Venezolana de Gerencia, 13(42), 309-318. Universidad del Zulia Maracaibo.

Nieves, L. Y., \& León, S. M. (2001). La gestión del conocimiento: una nueva perspectiva en la gerencia de las organizaciones. ACIMED, 9(2), 121-126.

Pallares, V. Z. (1997). La asociatividad empresarial: Una respuesta de los pequeños productores a la internacionalización de las economías. PRODES, 1- 20.

Pérez, A. J. (2004). Solución de problemas en empresas. Un enfoque sistémico.

Pico, P. G., y López D., M. de la F. (2006). Importancia de la actividad comercial de las empresas latinoamericanas en la transnacionalización e internacionalización. Revista Venezolana de Ciencias Sociales, 10(1), 69-85.

Pinkas, F. (2003) ¿Por qué fracasan las empresas? re atrincheramiento, reflotamiento y recuperación en entornos depresivos. Facultad de Ciencias Económicas y Administrativas, Universidad El Bosque.

Pinto, J. (2008). Sistema de Gestión de Competencias basado en capacidad y recursos y su relación con el sistema SECI de gestión del conocimiento, realizadas por las pequeñas empresas del Urola medio (España). Estudios Gerenciales, 23(105), 13-38.

Puerto,D.P. (2010). La globalización y el crecimiento empresarial a través de estrategias de internacionalización. Pensamiento y Gestión, (28) 171-195. Universidad del Norte.

Quero, L. (2008). Estrategias competitivas: Factor clave del desarrollo. Revista Negotium, 10(4), 36-49. En www.revistanegotium.org.ve

Revuelta, J., y Alonso, F. (2013). Presencia de las multilatinas en Europa. Tipología y Estrategia Empresarial. ICEI. WP, (03), 1-42.

Rincón, I. C. (2004). La administración en el nuevo entorno internacional. Revista Escuela de Administración y Negocios (EAN), (51), 17-21. Escuela de Administración de Negocios (EAN).

Rivera, E. (2014). Empresas multinacionales latinoamericanas. Los casos de Brasil y Chile. Serie Políticas Públicas y Transformación Productiva, (15). Corporación Andina de Fomento. 
Rivera, C. A. (2009). Colombia y sus opciones comerciales. Expo notas. Dirección de Asuntos Económicos de Analdex. XXI Congreso Nacional de Exportadores. Estrategia exportadora frente a la crisis económica: Como minimizar sus efectos. XXI Congreso Nacional de Exportadores. Expo Notas, (pp. 1-24).

Rodríguez, M. L. (1995). La gerencia interinstitucional. El liderazgo de espacios abiertos. Revista del CLAD Reforma y Democracia, (3), 1-8.

Sierra, J. H. (2003). La propiedad y el control en las decisiones de internacionalización de las empresas. Cuad. Adm. 16 (26), 99-128.

Varela, B. E. (2011). Políticas y estrategias en la gestión de EPM. (1 $1^{\mathrm{a}}$ ed.). Medellín. Ediciones de la Universidad del Valle, Universidad Libre de Cali.

Villarreal, L. O. (2005). La internacionalización de la empresa y la empresa multinacional: una revisión conceptual contemporánea. Cuadernos de Gestión, 5(2), 55-73.

Yákolev, P. (2013). Multilatinas: salto transfronterizo del negocio latinoamericano. Traducción del artículo publicado en la revista rusa ЛатинскаяАмерика. Iberoamérica, (6), 5-33. 
